\title{
THE ‘MOON MAPPING’ PROJECT TO PROMOTE COOPERATION BETWEEN STUDENTS OF ITALY AND CHINA
}

\author{
M. Scaioni ${ }^{\text {a, }{ }^{*} \text {, P. Giommi }}{ }^{\text {b }}$, M.T. Brunetti ${ }^{\text {c }}$, C. Carli ${ }^{\text {d, }}$ P. Cerroni ${ }^{\text {d }}$, G. Cremonese e, G. Forlani ${ }^{\text {f }}$, P. Gamba ${ }^{\text {g, }}$ M. Lavagna ${ }^{\text {h }}$,

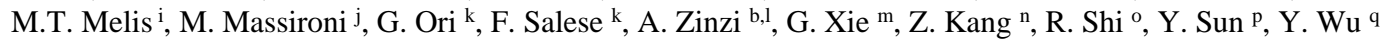 \\ a Dept. of Architecture, Built environment and Construction engineering (ABC), Politecnico di Milano \\ via Ponzio 31, 20133 Milano, Italy - marco.scaioni@polimi.it \\ b ASI Science Data Center, Italian Space Agency (ASI-ASDC), Roma, Italy - \{paolo.giommi,zinzi\}@asdc.asi.it \\ ${ }^{\mathrm{c}}$ Research Institute for Geo-Hydrological Protection - Italian National Research Council (IRPI-CNR) \\ via Madonna Alta, 126, 06128 Perugia, Italy - mariateresa.brunetti@irpi.cnr.it \\ ${ }^{\mathrm{d}}$ National Institute for Astrophysics, Institute for Space Astrophysics and Planetology (INAF-IAPS) \\ via fosso del cavaliere, 00133 Roma, Italy - \{cristian.carli,priscilla.cerroni\}@iaps.inaf.it \\ e National Institute for Astrophysics, Astronomical Observatory of Padua (INAF-OAPD), Padova, Italy \\ gabriele.cremonese@oapd.inaf.it \\ ${ }^{\mathrm{f}}$ Dept. of Civil, Environmental, Land Management Engineering and Architecture (DICATeA), University of Parma \\ Parco Area delle Scienze 181/A, 43124 Parma, Italy - gianfranco.forlani@unipr.it \\ ${ }^{g}$ Dept. of Electrical, Computer and Biomedical Engineering, University of Pavia, via Ferrata 5, 27100 Pavia, Italy \\ paolo.gamba@unipv.it \\ ${ }^{\text {h }}$ Dept. of Aerospace Science \& Technology, Politecnico di Milano, via La Masa 34, 20156 Milano, Italy \\ michelle.lavagna@polimi.it \\ ${ }^{i}$ Dept. of Chemical and Geological Sciences, University of Cagliari, via Trentino 51, 09127 Cagliari, Italy \\ titimelis@unica.it \\ j Dept. of Geosciences, University of Padua, via G. Gradenigo 6, 35131 Padova, Italy - matteo.massironi@unipd.it \\ ${ }^{\mathrm{k}}$ IRSPS - Dept. of Engineering and Geology, Università d'Annunzio, \\ 65127 Pescara, Italy\{ggori,francesco\}@irsps.unich.it \\ ${ }^{1}$ National Institute for Astrophysics, Astronomical Observatory of Rome (INAF-OAR), Roma, Italy \\ ${ }^{m}$ Center of Space Exploration, China Ministry of Education, Chongqing University, Chongqing, China \\ ${ }^{\mathrm{n}}$ Dept. of Remote Sensing and Geo-Information Engineering, School of Land Science and Technology \\ China University of Geosciences, Xueyuan Road 29, Haidian District, 100083 Beijing, China - zzkang@cugb.edu.cn \\ ${ }^{\circ}$ School of Geographic Sciences, East China Normal University, Shanghai, China \\ p Department of Computer Science and Technology, Tsinghua University, Beijing, China - syk@tsinghua.edu.cn \\ q School of Geographic and Oceanographic Sciences, Nanjing University \\ Xianlin Boulevard 163, Qixia District, Nanjing 210023, China - wu@nju.edu.cn
}

Commission VI, WG VI/3

KEY WORDS: Planetary mapping, Moon, Chang'E 1/2, Student Exchange, International Cooperation

\begin{abstract}
:
The research project 'Moon Mapping' has been established in 2014 between the Italian and Chinese Governments to promote cooperation and exchange between undergraduate students from both countries. The operational phase of the project started in early 2015, and will end in 2017, for a total length of three years. The main aim is to train new scholars to be able to work on different kinds of remotely-sensed data collected over the Moon surface by the Chinese space missions Chang'E-1/2. The project coordination has been assigned to the Italian Space Agency for the Italian side and to the Center of Space Exploration, China Ministry of Education, for the Chinese side. Several Chinese universities and Italian national research institutes and universities have been officially involved in this project. Six main research topics have been identified: (1) map of the solar wind ion; (2) geomorphological map of the Moon; (3) data preprocessing of Chang'E-1 mission; (4) map of element distribution; (5) establishment of 3D digital visualization system; and (6) compilation and publication of a tutorial on joint lunar mapping.
\end{abstract}

(*) corresponding author 


\section{GENESIS OF THE PROJECT}

On the basis of the great experience that Italy and China have achieved in space exploration over the last decades, the governments of both countries agreed to foster scientific and technological cooperation in this field. In addition, both countries would like to promote the exploitation of existing large archives of data sets, in particular the ones collected during the recent Chinese missions (Chang'E series) on the Moon (see Sect. 3). In this context, the education and the exchange of new generation scholars to be enrolled in space exploration and planetary sciences are seen as a crucial points in both countries.

According to these preliminary considerations, in 2012 the 'Joint Lunar Map Drawing Project by Chinese and Italian College Students,' also know as 'Moon Mapping Project' (MMP), was organized. The definitive signature was held in Rome on 23 January 2015 by the Italian Space Agency (ASI) Science Data Center (ASDC) director Paolo Giommi and the vice-president of the Chinese Center for Space Exploration (COSE) Gengxin Xie. The focus of this project is improve cooperation in space applications, to train new Italian and Chinese university undergraduate students to work on different kinds of remotely-sensed data collected over the Moon surface by the Chinese space missions Chang'E-1/2, to make easier student exchange between both countries, and to create scientific opportunities.

The operational phase started on Jan 2015 and is expected to complete at the end of 2017, for a total length of three-year activities. The project coordination has been assigned to the Italian Space Agency (ASI) for the Italian side and to COSE for the Chinese side. Several universities and national-level research institutes have been officially involved in the MMP on both countries, as presented in Table 1.

Six main research topics have been focused, as it is summarized in Table 2 (see Sect. 3). Each topic is coordinated by two institutions, one per each country. Students have been assigned to each topic to cooperate under the guide of supervisors. A schedule of mutual visits and workshops has been defined to help and promote exchanges along the three years of the project. With the exception of the first round meetings to set up the organization of the project and to prepare a common research plan, students are involved in project meetings so that they may consolidate the cooperation among them, especially between students from both countries. Further discussion on the student involvement, which may be considered as a key-point of the MMP, are given in next subsection 1.2.

The expected follow-up will consist on one side on the preparation of a new generation of young scholars to contribute to planetary science and space exploration. On the other, the MMP is expected to output scientific publications, a tutorial aimed at introducing people to work on planetary data, and a 3D Atlas of the Moon to display the results of the analysis obtained during the research activities.

Further information, documents and news can be found at the website http://solarsystem.asdc.asi.it/change.

\begin{tabular}{|c|l|c|c|}
\hline \multicolumn{2}{|l|}{ Project Topics } & \multicolumn{2}{c|}{ Topic Coordinators } \\
\cline { 3 - 4 } & Italy & China \\
\hline 1 & $\begin{array}{l}\text { Map of the solar wind } \\
\text { ion }\end{array}$ & ASDC & $\begin{array}{c}\text { Tsingua } \\
\text { University }\end{array}$ \\
\hline 2 & $\begin{array}{l}\text { Geomorphologicl map of } \\
\text { the Moon }\end{array}$ & $\begin{array}{c}\text { Politecnico } \\
\text { Milan }\end{array}$ & $\begin{array}{c}\text { China Univ. } \\
\text { Geosciences }\end{array}$ \\
\hline 3 & $\begin{array}{l}\text { Data preprocessing of } \\
\text { Chang'E 1 mission }\end{array}$ & INAF-IAPS & $\begin{array}{c}\text { East China } \\
\text { Normal Uni }\end{array}$ \\
\hline 4 & $\begin{array}{l}\text { Map of element } \\
\text { distribution }\end{array}$ & Univ. of & $\begin{array}{c}\text { Nanjing } \\
\text { University }\end{array}$ \\
\hline 5 & 3D visualization system & ASDC & $\begin{array}{c}\text { China Univ. } \\
\text { Geosciences }\end{array}$ \\
\hline 6 & $\begin{array}{l}\text { Tutorial and Atlas on } \\
\text { joint lunar mapping }\end{array}$ & $\begin{array}{l}\text { Chongqing } \\
\text { University }\end{array}$ & $\begin{array}{c}\text { University } \\
\text { d'Annunzio }\end{array}$ \\
\hline
\end{tabular}

Table 2. - Topics of 'Moon Mapping Project' and institutions leading research activities in Italy and China.

\begin{tabular}{|c|c|c|}
\hline Institution & Department & Location, Country \\
\hline Italian Space Agency (ASI) & ASI Science Data Centre (ASDC) & Rome, Italy \\
\hline \multirow{3}{*}{$\begin{array}{l}\text { National Inst. of Astrophysics } \\
\text { (INAF) }\end{array}$} & Astronomical Observatory of Rome (OAR) & \multirow{2}{*}{ Rome, Italy } \\
\hline & Institute for Space Astrophysics and Planetology (IAPS) & \\
\hline & Astronomical Observatory of Padua (OAPD) & Padua, Italy \\
\hline National Research Council (CNR) & Research Inst. for Geo-Hydrological Protection (IRPI) & Perugia, Italy \\
\hline \multirow{2}{*}{$\begin{array}{l}\text { Politecnico di Milano } \\
\text { (Technical University of Milan) }\end{array}$} & Dept. of Aerospace Science and Technology & \multirow[t]{2}{*}{ Milan, Italy } \\
\hline & Dept. Architecture, Built environment and Construction Engineering (ABC) & \\
\hline University of Cagliari & Dept. of Chemical and Geological Sciences & Cagliari, Italy \\
\hline University d'Annunzio & IRSPS - Dept. of Engineering and Geology & Pescara, Italy \\
\hline University of Padua & Dept. of Geosciences & Padua, Italy \\
\hline University of Parma & $\begin{array}{l}\text { Dept. of Civil, Environmental, Land Management Engineering and } \\
\text { Architecture (DICATeA) }\end{array}$ & Parma, Italy \\
\hline University of Pavia & Dept. of Electrical, Computer and Biomedical Engineering & Pavia, Italy \\
\hline China Ministry of Education & Center of Space Exploration (COSE), Chongqing University & Chongqing, China \\
\hline China University of Geosciences & $\begin{array}{l}\text { Dept. of Remote Sensing and Geo-Information Engineering, School of Land } \\
\text { Science and Technology }\end{array}$ & Beijing, China \\
\hline East China Normal University & School of Geographic Sciences & Shanghai, China \\
\hline Nanjing University & School of Geographic and Oceanographic Sciences & Nanjing, China \\
\hline Tsinghua University & Dept. of Computer Science and Technology & Beijing, China \\
\hline
\end{tabular}

Table 1. - Members of 'Moon Mapping Project' between Italy and China. 


\subsection{Involvement of students}

The core of 'Moon Mapping' project is represented by the central role of undergraduate students who are expected carry out the research activity under supervision of senior researches from universities and other institutions.

Students are assigned to specific Topics and Sub-Topics of the project. Since there is a parallel structure involving both Italian and Chinese project members, also students have a direct counterpart working on a related topic. This strict cooperation would like to give the chance to compare different approaches to the research work, and to improve the capability to accomplish scientific investigations thanks to the exchange of ideas.On the other hand, students have the chance to work together with expert scholars in the field of Moon exploration and planetary research, and can benefit from the experience of the international network related to the MMP.

Besides students who directly attend to the research activity, some dissemination actions are planned during the last year of the project. In particular, the organization of an international summer school on the processing techniques remote sensing data on the Moon is scheduled. This important activity will spread out the knowledge achieved during the MMP to a larger scenario of young scholars not only limited to Italy and China, but with the chance to involve students from other countries as well.

\section{DATA SETS FROM CHANG'E 1/2 MISSIONS}

The Chinese Lunar Exploration Program (CLEP), also known as the Chang'E (the Chinese Moon goddess), is an series of missions organized by the China National Space Administration (CNSA). The program incorporates lunar orbiters, landers, rovers and sample return spacecraft, launched using Long March rockets.

The first spacecraft of CLEP/Chang'E program, the Chang'E 1 (CE-1 - see Subsect. 2.1) lunar orbiter, was launched on 2007. A second orbiter, Chang'E 2 (CE-2), was launched on 2010 (see Subsect. 2.2). Chang'E 3 (CE-3), which includes a lander and a rover, was launched on 1 December 2013 and successfully soft-landed on Mare Ibridum on 14 December 2013. The 'Yutu' rover travelled $114 \mathrm{~m}$. CE-3 will be followed by a sample return mission, Chang'e 5 scheduled for 2017.

The 'Moon Mapping' project was initially focused to exploit data from CE-1 mission, but subsequently the availability of some data sets from CE-2 became possible. A part of the data sets from CE-1/CE-2 missions can be downloaded from the webpage http://moon.bao.ac.cn/ceweb/datasrv/dmsce1 .jsp.

\subsection{Chang'E 1}

On October 24, 2007, CE-1 was successfully launched from the Xichang Satellite Launch Center. Up until $1^{\text {st }}$ July 2008, CE-1 has accomplished lunar global data acquisition. CE-1 carries 8 instruments (CCD stereo camera, laser altimeter, Gamma Spectrometers, X-Ray Spectrometers, ow-Energy
Ion Detector, High-Energy Solar Particle Detector, Microwave Detector, Interferometer Spectrometer Imager) which have obtained about $1370 \mathrm{G}$ of data. These data have been published online at present, available for international planet researchers (see above).

CE-1 mission had four major goals:

1. Obtaining 3D reconstructions of the landforms and geological structures of the lunar surface, so as to provide a reference for planned future soft landings. The orbit of CE-1 around the Moon was designed to provide complete coverage, including areas near the north and south poles not covered by previous missions;

2. Analysing and mapping the abundance and distribution of various chemical elements on the lunar surface as part of an evaluation of potentially useful resources on the Moon. China hopes to extend the number of elements studied to 14 (K, Th, U, O, Si, Mg, Al, Ca, Te, $\mathrm{Ti}, \mathrm{Na}, \mathrm{Mn}, \mathrm{Cr}, \mathrm{La})$ compared to the 10 elements (K, U, $\mathrm{Th}, \mathrm{Fe}, \mathrm{Ti}, \mathrm{O}, \mathrm{Si}, \mathrm{Al}, \mathrm{Mg}, \mathrm{Ca}$ ) previously probed by NASA's Lunar Prospector;

3. Probing the features of the lunar soil and assessing its depth, as well as the amount of helium-3 $\left({ }^{3} \mathrm{He}\right)$ present; and

4. Probing the space environment between 40,000 km $400,000 \mathrm{~km}$ from the Earth, recording data on the solar wind and studying the impact of solar activity on the Earth and the Moon.

In addition, the lunar probe engineering system, composed of five major systems - the satellite system, the launch vehicle system, the launch site system, the monitoring and control system and the ground application system - accomplished five goals:

- Researching, developing and launching China's first lunar probe;

- Mastering the basic technology of placing satellites in lunar orbit;

- Conducting China's first scientific exploration of the Moon;

- Initially forming a lunar probe space engineering system; and

- Accumulating experience for the successive phases of China's lunar exploration program.

\subsection{Chang'E 2}

CE-2 is a Chinese unmanned lunar probe that was launched on 1 October 2010. It was a follow-up to the CE-1 lunar probe, which was launched in 2007. CE-2 was part of the first phase of the Chinese Lunar Exploration Program, and conducted research from a 100-kilometer-high lunar orbit in preparation for the December 2013 soft landing by the CE-3 lander and rover. CE-2 was similar in design to CE-1, although it featured some technical improvements, including a more advanced onboard camera. After completing its primary objective, the probe left lunar orbit for the EarthSun L2 Lagrangian point, to test the Chinese tracking and 
control network, making the China National Space Administration the third space agency after NASA and ESA to have visited this point. It entered orbit around L2 on 25 August 2011, and began transmitting data from its new position in September 2011. In April 2012, CE-2 departed L2 to begin an extended mission to the asteroid 4179 Toutatis, which it successfully flew by in December 2012. This success made China's CNSA the fourth space agency to directly explore asteroids, after NASA, Europe's ESA and Japan's JAXA. As of 2014, CE-2 has travelled over 100 million kilometres from Earth, and is conducting a long-term mission to verify China's deep-space tracking and control systems.

\section{PLANNED RESEARCH ACTIVITIES}

In this section the research content to be developed in each project Topic is briefly outline. The purpose here is not to draw a state-of-the-art on the use of CE-1/2 data for mapping different characteristics on the Moon, but just to focus on the activities that are strictly related to MMP. Some projects members have already developed their own studies that are not mentioned here, since they have been carried out independently from MMP.

\subsection{Topic 1}

The aim of Topic 1 is to produce a map of the solar wind ions on the basis of CE-1 data. Based on the lunar probe data and existing research results, the distribution of solar wind ion at critical moments will be drawn, by which to provide an interactive visualization approach for drawing low-energy solar ion flow distribution at arbitrary time, giving a real-time display of solar wind ion flow and its direction.

The workplan of this Topic is as follows. In a first stage, a sample of data from CE-1 Solar Wind Ion Detector are delivered (664 files collected from 26 Nov 2007 to 31 Dec 2007). Data are organized in a database hosted on a server at ASDC. File format are transformed to be ready to use. The following two data post-processing/analysis steps have been started:

1. Spacecraft velocity evaluation;

2. Sun Incidence angle evaluation.

Next steps in data post-processing will be:

1. Merging of SWIDA and SWIDB (time synchronization);

2. Evaluation of solar wind parameters by SWIDA/B data fit;

3. Production of Multidimensional solar wind maps;

4. Evaluation of Earth/Sun magnetic fields; and

5. Correction of the magnetic field effects.

\subsection{Topic 2}

This Topic is focused on the analysis of Moon geomorphology and the production of geomorphological maps representing different aspects. It is organized in three Sub-topics. a. Sub-topics 2.1: is devoted to the extraction of geometrical features for characterization of the impact structures and proximal ejecta, development of crater morphology and degradation;

b. Sub-topic 2.2: focuses to the extraction and characterization of landslides on the Moon surface, in particular into the impact craters. Analysis of landslide triggering process and impact-related effects will be investigated as well; and

c. Sub-topic 2.3: has the aim of doing research and characterization of lava-tubes below the Moon surface and their relations with sinuous rilles.

Impact craters are one of the most important geological processes in the understanding of the formation and evolution of our Solar System. They have been observed on planets and small bodies' surfaces, and therefore they kept the record of the cumulative effects of subsequent impacts, volcanic emplacements, tectonics, and so on. The MMP team has developed a valuable expertise on studying the impact craters starting from the DTM generation using stereo images and proceeding with the morphometric analysis. The morphometric characterization of the craters allows estimating the slope, the openness, the profile and plan curvature, and calculating the different geometrical planes. Furthermore the analysis of the impact process by means of the so-called shock physics codes has been started. In particular, the iSale hydrocode is adopted that relies on elasto-plastic constitutive models, fragmentation models, a number of EoS, multiple materials and a porosity compaction model.

In line with the consolidated cooperation with China University of Geosciences on geometrical feature extraction from Moon images (Kang et al., 2015), the team at University of Pavia is currently working on the extraction of hints to craters and ridges using both 2D and 3D information from the stereo cameras of CE- 1 and CE-2. The research work includes the definition of advanced techniques for the extraction of circular and linear geometrical features and the combination via feature level fusion of multiple hints for the detection of objects of interest, according to the procedure graphically described in Figure 3.

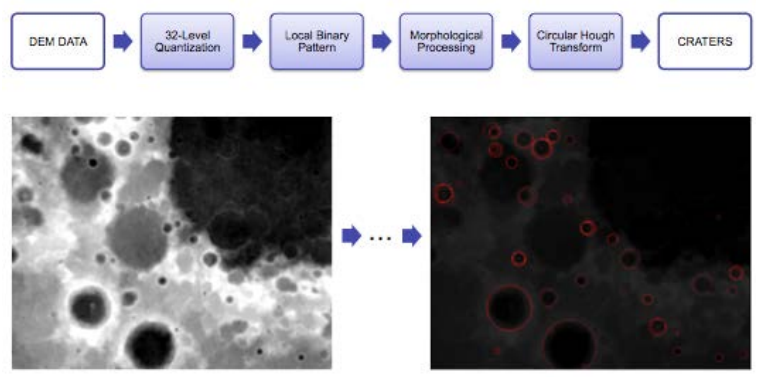

Figure 3. - Crater extraction procedure scheme developed by University of Pavia. 
Results, although preliminary, show potential in the approach, that will be further optimized to deal with large images, with the aim to be useful for finer resolution data on wide portions of the lunar surface.

A working group (Politecnico di Milano-ABC, CNR-IRPI, University of Cagliari, INAF-OAR and INAF-OAPD) has been established to study large rock slides inside impact craters and to prepare a landslide inventory to be included in the Atlas (see Subsect. 3.6). So far, image from WAC (Wide Angle Camera) and DEM from LROC (NASA) at $100 \mathrm{~m} / \mathrm{px}$ have been used to visually recognize 60 landslides in impact craters (Brunetti et al., 2015).

On the other hand, the need for a more objective automatic method is required in order to make the recognition process independent from the subjective interpretation and to carry out an exhaustive search (see Guzzetti et al., 2012; Scaioni et al. 2014). In Mahanti et al. (2014) the Chebyshev polynomials have been applied to interpolate crater crosssectional profiles in order to obtain a parametric characterization useful for classification into different morphological shapes. Indeed, besides some important mathematical properties (e.g., orthogonality) that distinguish Chebyshev polynomials from other approximating functions, the lower order coefficients are correlated to some geomorphological characteristic of the crater and its surroundings. As an example, the zero order coefficient represents the average ground surface elevation, the $1^{\text {st }}$ order is related with the local topographic slope and $2^{\text {nd }}$ with the crater depth. The purpose of the interpolation with the Chebyshev polynomials is to model each crater with a theoretical shape. An example of interpolation is shown in Figure 4. The use of such polynomial approximations for detecting post-impact degradation processes in lunar craters has been already proposed in Mahanti et al. (2015). Here the analysis of odd coefficients of Chebyshev polynomials has been applied to detect asymmetries in the crater profile. The concept that is pursued in this subtopic is to recognize landslides by analyzing the discrepancy between the actual crater cross-sectional profiles and the theoretical ones (Yordanov et al., 2016). Four cross-sections are considered per each crater. Deviations from the theoretical shape can be detected through the analysis of the Chebyshev coefficients so that the landslide is automatically identified. Whilst the LROC-DEM has been used for the initial set up of the methodology, data from CE-2 will be also considered in a successive stage, due to similar spatial resolution. Indeed, the acquired stereo images from the equipped charge-coupleddevice (CCD) camera can reach a resolution of $7 \mathrm{~m}$ from CE2 circular orbit (reaching a minimum distance from ground of approx. $100 \mathrm{~km}$ ), and $1.5 \mathrm{~m}$ around the perilune (minimum distance from ground of approx. $15 \mathrm{~km}$ ), see Zhao et al. (2011).

Further studies will include: the measurement of the landslide volume; the analysis of relationships between landslides and characteristics of the hosting craters as well as the surrounding terrain; the lithological and mineralogical characterization of surfaces using multispectral data acquired by the IIM data from CE-1.
Another working group (CNR-IRPI, University of Cagliari) will focus on the research and characterization of lava-tubes below the Moon surface. Recent planetary missions have made available large amounts of remote sensing data. Among the many interesting results obtained from the analysis of high-resolution images is the detection of lava tubes on Mars and on the Moon (Haruyama et al., 2009). These caves, also present on Earth, originate from cooling and the subsequent consolidation of the outermost part of very fluid lava flows. In the harsh lunar environment, lava tubes can provide a natural shelter. Detection and characterization of lava tubes and their relations with sinuous rilles will use data available from the multispectral sensor IIM and the CCD.

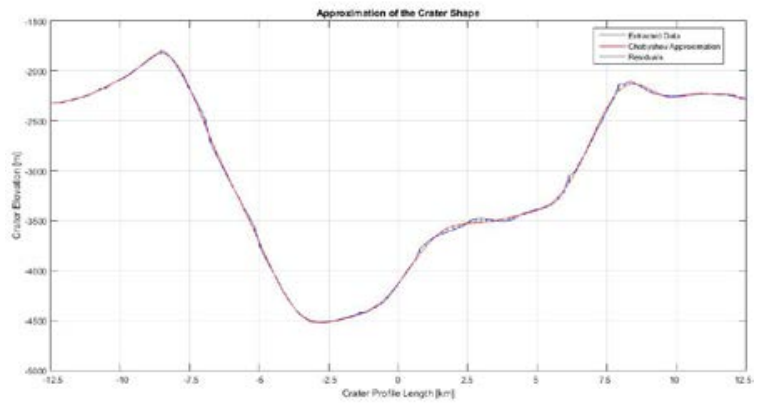

Figure 4. - Example of interpolation of a lunar crater crosssectional profile using Chebyshev polynomials.

\subsection{Topic 3}

Topic 3 has the aim of investigating calibration and preprocessing procedures for the sensors in the CE-1 payload. Prior to data analysis and application, preprocessing on the remotely sensed raw data is necessary for correcting distortion due to the characteristics of imaging systems and imaging conditions. It normally precedes further manipulation and analysis of the image data to extract specific information. The pre-processing of data is a crucial step in the remote sensing analytical workflow, and is often the most time consuming and costly.

Since the CE-1 ended about seven years ago, several research groups have already started working on data preprocessing. $\mathrm{Hu}$ et al. (2013) have coped with the calibration of laser altimetry data, obtaining significant improvement on the quality of the final DEM. Wu et al. (2013) have dealt with the photometric correction and in-flight calibration of Interference Imaging Spectrometer (IIM) data onboard CE1. In the literature, several experiences about calibration of other sensors during space missions are reported. For example, Haruyama et al. (2008) have worked on the radiometric calibration of digital camera adopted during SELENE mission.

Whilst other Topics of MMP are focused to output specific products, Topic 3 has a more general purpose and it's expected to contribute to those aspects of sensor data processing that may be required in other Topics of the 
project. In Figure 5, the pre-processing workflow of IIM data implemented during MMP is shown.

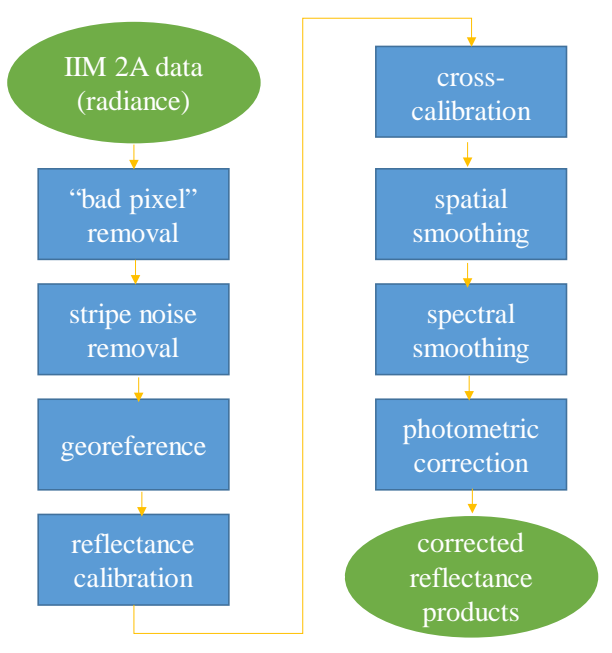

Figure 5. - IIM pre-processing workflow.

\subsection{Topic 4}

The aim of Topic 4 is to output a map of element distribution on the Moon surface on the basis of data acquired by the Interference Imaging Spectrometer (IIM) onboard CE-1 (Wu et al., 2010). IIM sensor covers a spectral range (0.48-0.96 $\mu \mathrm{m})$ that can be used to retrieve information about the differences of lunar surface composition. Indeed, reflectance spectra may offer important information on mineralogy and lithology. Starting from the experiences of Wu et al. (2010; 2013), the calibrated data acquired by IIM sensor will be used for the spectral characterization of the surfaces in terms of elements distribution, mineralogical composition and lithologic characterization. Both the indirect approach based on spectral parameters (see, e.g., Lucey et al., 2000), and the direct indication retrievable from the $0.9 \mu \mathrm{m}(\mathrm{Fe} 2+$ bearing silicates) absorption can be applied (even if IIM spectral range cannot investigate completely this absorption with respect to longer wavelength sensors). Moreover, by correlating spectral analysis of IIM data with other hyperspectral images covering a larger wavelength range (i.e., M3 data from sensor on onboard Indian satellite Chandrayaan-1 - see, e.g., Pieters et al., 2011), it will be possible to extrapolate more mineralogical indication from IIM data set. Laboratory spectral analysis on terrestrial analogues (e.g., Serventi et al., 2014), as well as lunar meteorites or lunar samples, can be useful to define these spectral relationships (e.g. Lucey et al., 2000).

At this first phase the IIM data are obtainable through the MATISSE web-tool (see Subsect. 3.5). The data are georeferenced and the full set of 26 bands, ranging from $522.37-918,109 \mathrm{~nm}$, is available.

As described in subsection 2.2, the IIM data will be also used for a specific lithological analysis of the landslides in the larger craters. In Figure 6, a preliminary processing result of these data is shown: the surface of this crater (diameter 7.5 $\mathrm{km}$ ) presents specific spectral anomalies.

The results of the research activities in Topic 4 will be integrated with photo-interpretation and classification of the main elements as volcanic morphologies and craters to support the evolution and dynamics of these morphologies. It is also planned to compare the processed data with existing spectral imagery as Clementine UVVIS camera bands and M3 products.

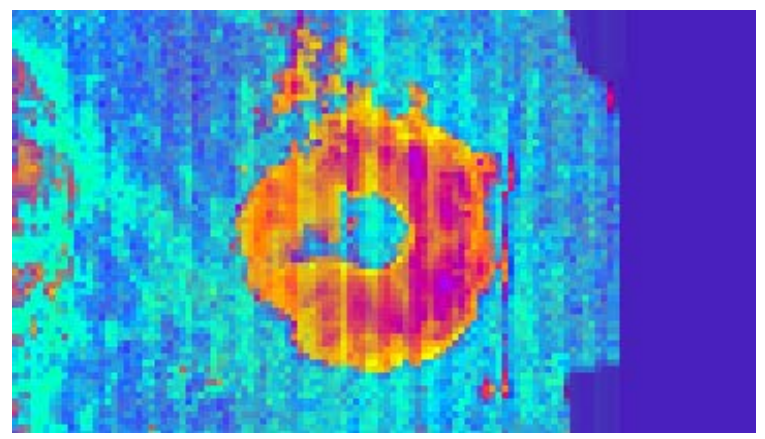

Figure 6. - The visualization of a lunar crater generated with a decorrelated stretching of a color composite using CE-1 IIM.

\subsection{Topic 5}

This Topic, devoted to the establishment of a 3D visualization system, would likely interact with the majority of the other Topics as, thanks to advanced visualization procedures, data analysis would boost with a great impact on scientific return.

At the present time the Italian side of the Moon Mapping project is focused in adding to the MATISSE web-tool (http://tools.asdc.asi.it/matisse.jsp - Zinzi et al., 2016) the data acquired by the Chinese missions to the Moon.

Very recently, all the CE-1 observations acquired with the visible CCD camera and the VIS/IR IIM spectrometer have been made available to the MMP users using MATISSE.

Furthermore, higher-order products of both CE-1 and CE-2 are already present in the MATISSE database, including DEM and orthoimages.

Using MATISSE it is possible to retrieve spatial data on the basis of a geographical query and to select one or more observations in order to either display a single observation or compute high-order products (i.e., mosaics, ratios, differences, RGB images) on the basis of available observations. The corresponding outputs can be managed by means of popular GIS desktop software packages (with GeoTIFF and ENVI format outputs) or using the advanced 3D tools provided by Paraview files (Fig. 7).

In the next future the number of observations available would likely rise, including also Solar Wind Ion Detector (SWID) and CE-2 data set, thus allowing the full exploitation of the available archives. 


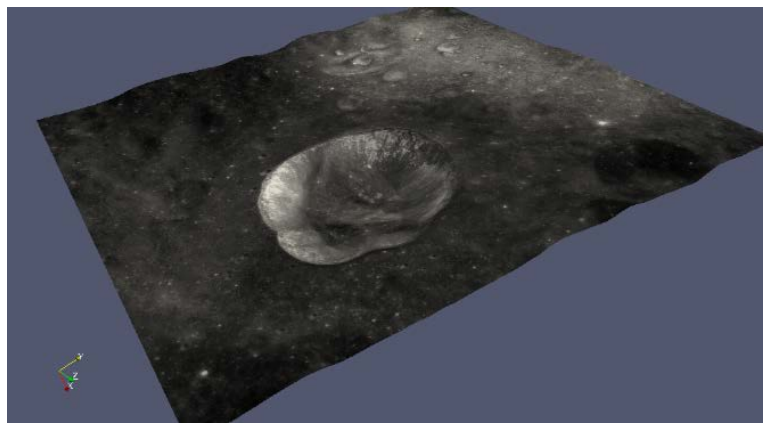

Figure 7. - The 3D visualization of a lunar crater generated using CE-2 high resolution ortophoto and NASA DEM (details in Zinzi et al., 2016).

\subsection{Topic 6}

IRSPS will edit a Moon Atlas dealing with the geological characterization of a number of sites in proximity of the maximum illumination areas of the lunar poles. The Atlas dataset will be based on CE-1 and CE-2 images and data. The Atlas will use as starting point the work by Mazarico et al. (2011) and will concentrate on both lunar poles. These areas bear strong scientific interest such as the history of volatile (Chin et al., 2007), but, also, they are interesting for the human exploration.

Data from these areas are critical for the planning and operations of future surface mission since:

- these sunlit areas could be prime locations for the establishment of solar photovoltaic arrays, and, consequently, they are rather efficient in power generation;

- sunlit area are thermally benign, the surface temperatures at the lunar equator and mid-latitudes depend almost entirely upon incident solar illumination, whereas the surface temperature of the permanent or quasi-permanent lit areas is nearly constant (Mazarico et al., 2011) facilitating the thermal design of surface habitats and equipment;

- lunar regions from about $80^{\circ}$ to the pole are of particular interest due to the possible presence of volatiles (Watson et al., 1961; Paige et al., 2010), especially in the permanent shadow regions (PSRs) seen as potential cold traps of volatiles (Nozette et al., 1996; Feldman et al., 2000; Bussey et al., 2003; Mitrofanov et al., 2010a,b).

\section{CONCLUSIONS AND PROSPECTS}

This paper has outlined the 'Moon Mapping Project' between university and research institutes of Italy and China. The project has seen its kick-off on 2015 and is going to develop up to the scheduled end on 2017. The focus of the project is to promote cooperation between both countries in space applications and science. The main feature that characterize
'Moon Mapping Project' is the strong student-oriented character. The research activity are promoted to actively involve undergraduate students and to make easier exchanges between both countries. Using the data of China's lunar exploration, undergraduates from both countries work together to map the moon in three dimension, and share the cooperation achievements globally.

The cooperation between Italy and China is expected to deepen cooperation in the field of science and education. The main anticipated achievement of the cooperation is the joint publication of a textbook on lunar remote-sensing image processing. The textbook will be published in English and will be used either in relevant university classes and also as a guide-book in museums.

\section{Acknowledgements}

The main acknowledgements go to the Italian Space Agency and the Center of Space Exploration of China Ministry of Education which have promoted, funded and supported this project. Acknowledgements also go to all administrations of Italian and Chinese universities and research centres involved in 'Moon Mapping Project,' since they are actively contributing to its positive development. The authors would like to sincerely thank the Embassy of Italy in Beijing and especially the Science \& Technology Councellor (Prof. Plinio Innocenzi) who promoted this project and gave a great support during the preparation stage. The authors would also thank all colleagues that have been supporting in some way the research or the organizational activities (travels, workshops, etc.). Eventually, many thanks go to students, who are playing a primary role in this project.

\section{REFERENCES}

\section{References from journals:}

Brunetti, M.T., Xiao, Z., Komatsu, G., Peruccacci, S., Guzzetti, F., 2015. Large rockslides in impact craters on the Moon and Mercury. Icarus, 260: 289-300.

Bussey, D.B.J., Lucey, P.G., Steutel, D., Robinson, M.S., Spudis, P.D., Edwards, K.D., 2003. Permanent shadow in simple craters near the lunar poles. Geophysical Research Letters, Vol. 30, paper No. 1278.

Chin, G., and other 16, 2007. Lunar Reconnaissance Orbiter overview: The instrument suite and mission. Space Science Review, Vol. 129, 29 pages.

Feldman, W., Lawrence, D., Elphic, R., Barraclough, B., Maurice, S., Genetay, I., Binder, A., 2000. Polar hydrogen deposits on the Moon. Journal of Geophysical Research, Vol. 105: 4175-4195.

Guzzetti, F., Mondini, A.C., Cardinali, M., Fiorucci, F., Santangelo, M., Chang, K.T., 2012. Landslide inventory maps: new tools for and old problem. Earth Science Review, Vol. 112(1-2): 42-66.

Haruyama, J., and 11 others, 2008. Planned radiometrically calibrated and geometrically corrected products of lunar high-resolution Terrain Camera on SELENE. Advances in Space Research, Vol. 42: 310-316. 
Haruyama, J., and 13 others, 2009. Possible lunar lava tube skylight observed by SELENE cameras. Geophysical Research Letters, Vol. 36(21), paper no. L21206.

Hu, W., Di, K., Liu, Z., Ping, J., 2013. A new lunar global DEM derived from Chang'E-1 Laser Altimeter data based on crossover adjustment with local topographic constraint. Planetary and Space Science, Vol. 87: 173-182.

Jin, S., Arivazhagan, S., Araki, H., 2013. New results and questions of lunar exploration from SELENE, Chang'E-1, Chandrayaan-1 and LRO/LCROSS. Advances in Space Research, Vol. 52: 285-305.

Kang, Z., Luo, Z., Hu, T., Gamba, P., 2015. Automatic extraction and identify-cation of lunar impact craters based on optical data and DEMs acquired by the Chang'E satellites. IEEE Journal of Selected Topics in Applied Earth Observation and Remote Sensing, Vol. 8(10): 4751-4761.

Lucey, P.G., Blewett, D.T., Jolliff, B.L., 2000. Lunar iron and titanium abundance algorithms based on final processing of Clementine ultraviolet-visible images. Journal of Geophysical Research, Vol. 105(E8): 20,297-20,305.

Mahanti, P., Robinson, M.S., Humm, D.C., Stopar, J.D., 2014. A standardized approach for quantitative characterization of impact crater topography. Icarus, Vol. 241: 114-129.

Mazarico, E., Neumann, G.A., Smith, D.E., Zuber, M.T., Torrence, M.H., 2011. Illumination conditions of the lunar polar regions using LOLA topography. Icarus, Vol. 211: 1066-1081.

Mitrofanov, I.G., and other 28, 2010a. Testing for hydrogen in permanently shadowed regions at the lunar south pole with the lunar exploration neutron detector on board LRO. Science, Vol. 330: 483-486.

Mitrofanov, I.G., and other 35, 2010b. Lunar exploration neutron detector for the NASA Lunar Reconnaissance Orbiter. Space Science Review, Vol. 150: 183-207.

Nozette, S., Lichtenberg, C.L., Spudis, P., Bonner, R., Ort, W., Malaret, E., Robinson, M., Shoemaker, E.M., 1996. The Clementine bistatic radar experiment. Science, Vol. 274: 1495-1498.

Paige, D.A. and other 26, 2010. Diviner lunar radiometer observations of cold traps in the Moon's south polar region. Science, Vol. 330: 479-482.

Pieters, and other 26, 2011. Mg-spinel lithology: A new rock type on the lunar farside. Journal of Geophysical Research, Vol. 116., paper No. E00G08.

Scaioni, M., Longoni, L., Melillo, V., Papini, M., 2014. Remote Sensing for Landslide Investigations: An Overview of Recent Achievements and Perspectives. Remote Sensing, Vol. 6(10): 9600-9652.

Sun, H., Dai, S., Yang, J., Wu, J., Jiang, J., 2005. Scientific objectives and payloads of Chang'E-1 lunar satellite. Journal of Earth System Science, Vol. 114(6): 789-794.

Watson, K., Murray, B.C., Brown, H., 1961. The behavior of volatiles on the lunar surface. Journal of Geophysical Research, Vol. 66: 3033-3045.

$\mathrm{Wu}, \mathrm{Y}$., and other 9, 2010. A preliminary experience in the use of Chang'E-1 IIM data. Planetary and Space Science, Vol. 58(14-15): 1922-1933.
Wu, Y., Besse, S., Li, J.-Y., Combe, J.-P., Wang, Z., Zhou, X., Wang, C., 2013. Photometric correction and in-flight calibration of Chang' E-1 Interference Imaging Spectrometer (IIM) data. Icarus, Vol. 222: 283-295.

Zhao, B., Yang, J., Wen, D., Gao, W., Chang, L., Song, Z., Xue, B., Zhao, W., 2011. Overall scheme and on-orbit images of Chang'E-2 lunar satellite CCD stereo camera. Science China - Technological Sciences, Vol 54: 2237-2242.

Zinzi, A., Capria, M.T., Palomba, E., Giommi, P., Antonelli, L.A., 2016. MATISSE: A novel tool to access, visualize and analyse data from planetary exploration missions. Astronomy and Computing, Vol. 15: 16-28.

\section{References from books:}

Serventi, G., Carli, C., Sgavetti, M., 2014. Laboratory Analysis (Reflectance Spectroscopy) of Terrestrial Analogues. Encyclopedia of Lunar Science. Springer International Publishing Switzerland, pp. 1-9.

\section{References from Other Literature:}

Mahanti, P., Robinson, M.S., Thompson, T.J., 2015. Characterization of lunar crater wall slumping from Chebyshev approximation of lunar crater shapes. In: Proc. "Annual Meeting of the Lunar Exploration Analysis Group (LEAG),” 20-22 Oct. 2015, Columbia, Maryland, U.S., paper No. 2081.

Yordanov, V., Scaioni, M., Brunetti, M.T., Melis, M.T., Zinzi, A., Giommi, P., 2016. Mapping Landslides in Lunar Impact Craters Using Chebyshev Polynomials and DEM's. Int. Arch. Photogramm. Remote Sens. Spatial Inf. Sci., Vol. XLI-VI Prague, Czech Republic, 8 pages. 